\title{
APT CCDTL AND CCL THERMAL/MECHANICAL ANALYSIS *
}

\author{
D. Christiansen, P. Smith, George Spalek \\ General Atomics, San Diego, CA 92186 , USA
}

\begin{abstract}
Design automation techniques are being developed to facilitate the design of the normal conducting CoupledCavity Drift Tube LINAC (CCDTL) and Coupled-Cavity LINAC (CCL) for the Accelerator Production of Tritium (APT). The cavity geometry is generated from automated RF design codes and fed directly into a 3-D multiphysics code which calculates the RF heat loads and cavity distortions due to the heat loads. The resulting frequency change in the cavity is determined by the Slater perturbation formulation. Cooling is located to minimize these distortions. The application and their status as applied to the APT CCL will be discussed. In particular, the use of specific codes to reduce the peak thermal stress around the coupling slots in these cw (continuous wave) RF cavities will be presented.
\end{abstract}

\section{INTRODUCTION}

An automation code has been developed that solve for CCL dimensions of normal conducting accelerating cavities (AC) and coupling cavities (CC). The code sets up input files for other codes to solve the electromagnetic, thermal, and structural problems associated with the design. Specific codes used in this process are the Coupling Cavity Tuning Code (CCT) [1], HFSS ${ }^{\circledast}$, and ANSYS $^{\oplus}$. Section 2 discusses the use of CCT and HFSS. Section 3 discusses the use of ANSYS and provides the status of development. Section 4 provides examples of stress analysis results.

\section{USE OF CCT TO DEFINE GEOMETRY}

The function of CCT is to solve for the dimensions of the $\mathrm{AC}$, the $\mathrm{CC}$, and the separation distance between the $\mathrm{AC}$ and $\mathrm{CC}$, such that the combined structure achieves specified design frequencies and coupling, k. CCT obtains a self-consistent solution in which the structure is tuned to the design frequencies and the effects of the coupling slot between the $\mathrm{AC}$ and $\mathrm{CC}$ are taken into account.

The CCT code controls the 2-D axisymmetric code, CCLFISH [2]. CCLFISH performs RF calculations for an individual cavity without a slot and tunes the cavity to an estimated target frequency, which accounts for the frequency shifts in the $\mathrm{AC}$ and $\mathrm{CC}$ caused by the coupling slot. The effects of the slot are modeled in CCT by means of semi-empirical equations [1]. These equations contain empirical constants called "A factors" that are used to adjust the theoretical equations to match experiment.

\footnotetext{
* Work supported by the APT project, U.S. DOE contract DE-AC04-96AL89607
}

A procedure has been developed [2] by which one can iterate between CCT, which establishes a trial design, and HFSS, a 3-D electromagnetic code, which is used to refine the estimates of the A factors. In this way, the combined 2-D and 3-D methods converge to a design much faster than if a 3-D code were used alone.

CCT prepares an interface text file containing geometrical parameter definitions. This file is read automatically by ANSYS while it is processing another input file that sets up and solves the electromagnetic, thermal, and structural problems. A few lines of this interface file are shown below as an example.

$$
\begin{aligned}
& \text { platelen }=2.319 \\
& \text { acdepth }=2.019 \\
& \text { acdiam }=11.621 \\
& \text { acrco }=1.569 \\
& \text { acrci }=0.472
\end{aligned}
$$

The names on the left are parameters used by ANSYS to define the 3-D solid geometry of the coupled CCL cavities.

\section{ANSYS - 3D ANALYSIS}

ANSYS is a general purpose finite element code that can solve a variety of "multiphysics" problems including the coupled electromagnetic, thermal, and structural problem described herein. This code has been tested with simplified 3-D models (not containing explicit coolant channels) to perform thermal-mechanical design analysis of the cavities. This analysis consists of

1. an electromagnetic analysis to calculate frequency, fields, and surface heat fluxes;

2. a thermal analysis to convert surface heat fluxes to internal solid temperatures;

3. a stress analysis to convert temperatures to displacements and stresses;

4. a calculation of the effect of the displaced boudary on the frequency shift using Slater perturbation theorem.

\subsection{Logic of Coupled Multi-physics Analysis}

Details of the logic for performing the coupled analysis are given below.

1. Read the geometrical input data from the CCT output file.

2. Construct a solid model of the coupled cavity geometry, including the $\mathrm{AC}, \mathrm{CC}$, and the coupling slot. The model consists of $1 / 2$ an accelerating cavity (sliced by a vertical plane parallel to the beam axis for symmetry) and two $1 / 4$ coupling cavities (sliced by the same symmetry plane as well as additional symmetry planes perpendicular to the beam axis. 
3. Construct a solid model of the copper structure surrounding the cavities by subtracting the cavities from a copper blank.

4. Model the cavities with high frequency electromagnetic (HF-emag) elements. Apply "electric wall" boundary conditions to the cavity model and "magnetic wall" boundary conditions to the $\mathrm{CC}$ ends and solve the HF-emag eigenvalue problem to obtain the accelerating $\pi / 2$ mode frequencies and the shapes of the magnetic and electric fields.

5. Calculate the average electric field along the beam axis from the fields of the HF-emag eigenvector, and scale the eigenvector fields so that the average electric field is equal to the value specified by the output of the Parmila (beam dynamics) code.

6. Search for elements on the metal surfaces of the cavity. For each such element, identify the face on the metal surface. For that face, convert the magnetic field values at the surface nodes into surface heat fluxes (caused by skin-effect currents) using the equation

$$
q=\frac{H^{2}}{2} \sqrt{\pi \mu_{0} f \rho}
$$

where $\mathrm{q}$ is the heat flux $\left(\mathrm{W} / \mathrm{m}^{2}\right), \mathrm{H}$ is the magnetic field $(\mathrm{A} / \mathrm{m}), \mu_{0}$ is the permeability of free space $\left(4 \pi 10^{7}\right)$, $\mathrm{f}$ is the frequency $(\mathrm{Hz})$, and $\rho$ is the bulk electrical resistivity of the copper (ohm-m). The quantity in the radical is the surface film resistance in ohm.

7. Switch to the finite element model of the metal structure. Apply cooling boundary conditions to the structure and the surface heat fluxes and solve for the internal metal temperatures.

8. Use the metal temperatures as thermal loads for a structural problem. Apply structural boundary conditions, including node tying constraints on symmetry surfaces, and solve for the stresses displacements.

9. Using a method similar to that for applying the heat fluxes, translate nodal displacements to the surfaces of the cavity elements.

10. Re-open the results file for the electromagnetic analysis of the cavity and determine the electric and magnetic fields at surface nodes corresponding to the surface displacements.

11. Calculate the frequency shift caused by the surface displacements acting against the fields using Slater perturbation theory,

$$
\frac{\Delta \mathrm{f}}{\mathrm{f}}=\int_{\mathrm{v}} \frac{\varepsilon_{0} \mathrm{E}^{2}-\mu_{0} \mathrm{H}^{2}}{4 \mathrm{U}} \mathrm{dV}
$$

where $f$ is the frequency, $\varepsilon_{0}$ is the permitivity of free space, $\mathrm{E}$ is the electric field $(\mathrm{V} / \mathrm{m}), \mathrm{U}$ is the total electric and magnetic energy in the cavity and $\mathrm{V}$ represents the volume of the displaced region adjacent to the surface.

\subsection{Status of Development}

All of the above steps have been tested in a proof-ofprinciple analysis in a simplified test problem. Steps 1-8, the thermal stress analysis portion, have been automated through the CCT interface and applied to a design assessment of the CCL (see the example is below). Steps 9-11 require a little more work before they are applied to a practical design problem. Tasks remaining to be performed are discussed below.

1. Explicit modeling of coolant channels has been performed on a trial basis using 2-D analysis with axisymmetric coolant channels. Since coupling slot cooling and distortions and coolant channel routing are design issues, a method must be worked out for explicitly modeling 3-D coolant channels in the metal structure and for optimizing their sizes and configurations. It is expected that this will be an automated $\mathrm{CAD}$ interface to define the coolant channel geometry and another program to perform solid modeling operations in ANSYS and to relate 1-D elements that model pipe flow and heat transport to heat fluxes on the 3-D channel surfaces.

2. Structural boundary conditions with node tying constraints needs to be implemented. Present boundary conditions are simplified.

3. The Slater perturbation procedure needs to be automated. Presently it is done by manual manipulation of files and an external Fortran program.

4. Further work is needed to quantify accuracy vs. mesh refinement. Even without coolant channels, the meshes being used generate problems that are quite large, around 500,000 degrees of freedom.

5. The work needs to be extended to model CCDTL cavities, which are larger and more complicated, with resulting limitations on element size.

\section{EXAMPLE OF STRESS ANALYSIS}

Following are a few pictures that illustrate a thermal stress analysis of a cavity. This analysis was of a cavity at APT CCL segment 322, the CCL segment with the highest power losses. To simplify this analysis, constant temperature boundary conditions were applied to the cooled surfaces on the brazed cavity plate interfaces. The coupling slot was not cooled. The results indicate that thermal stresses are an issue at the root of the slot, where the magnetic fields are concentrated, so routing of the cooling channels to the slot area is required. 


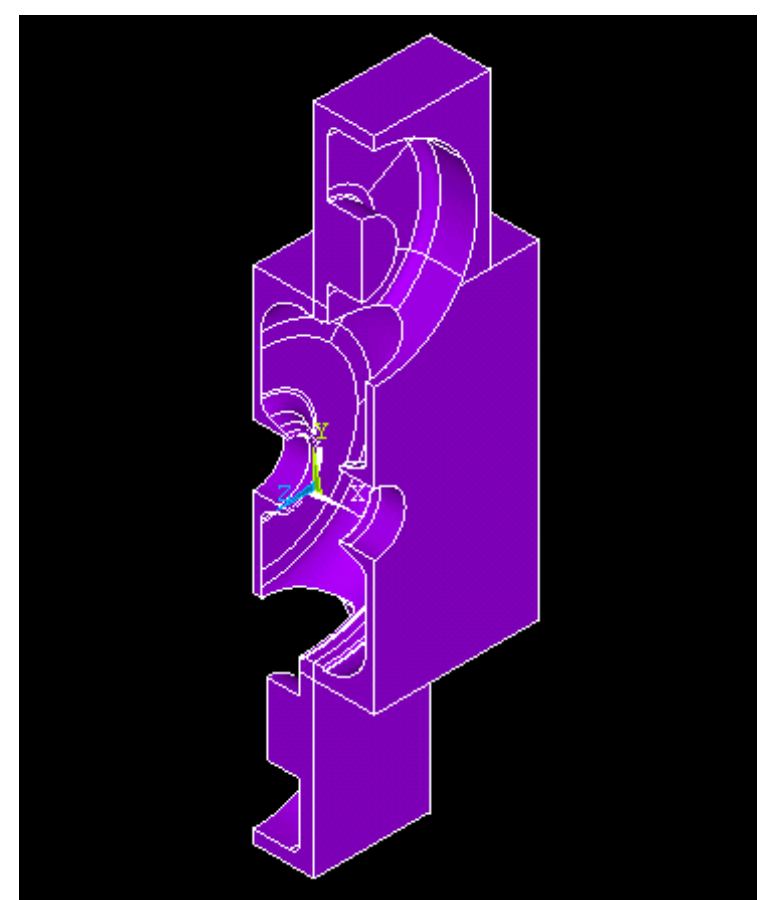

Figure 1: Solid model of copper structure

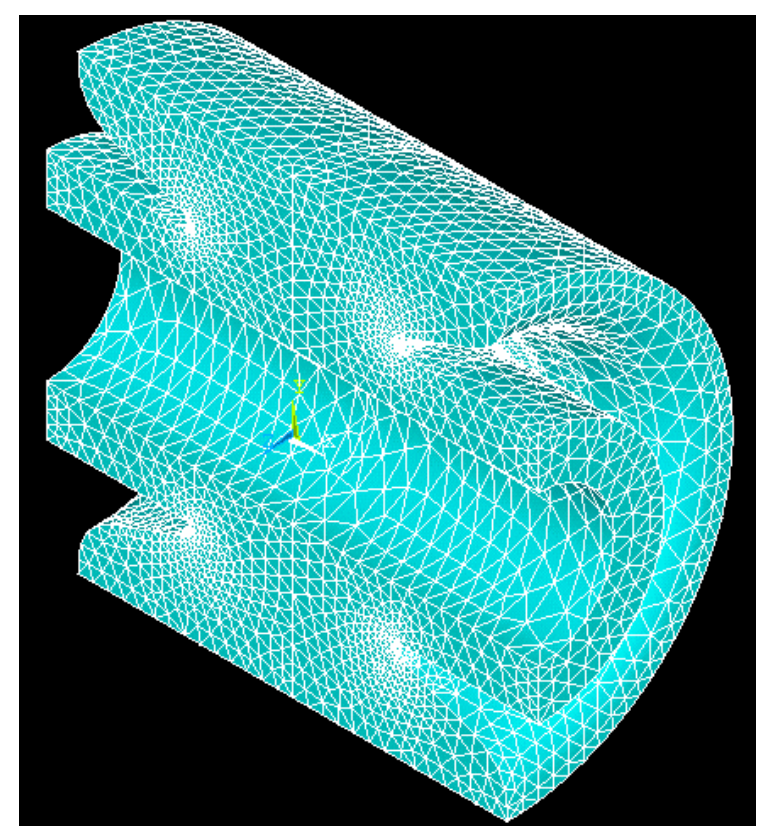

Figure 2: Portion of graded mesh of cavity

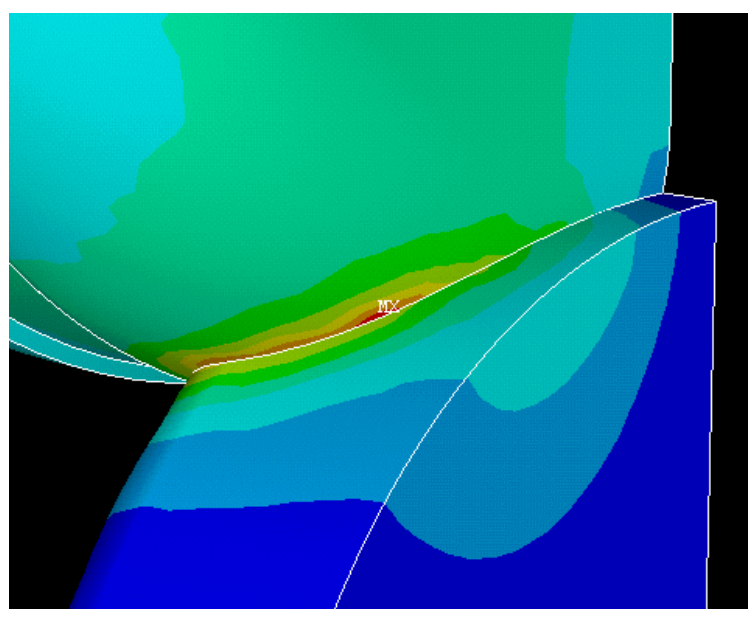

Figure 3: Peak of magnetic field around slot (cavity)

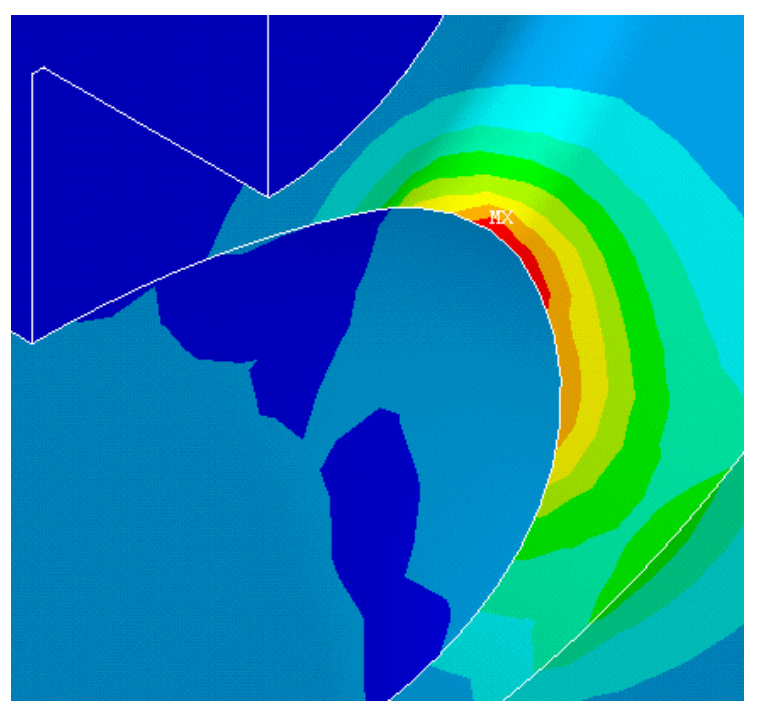

Figure 4: Von Mises stresses around slot (copper)

\section{REFERENCES}

[1] P. D. Smith, "CCT, a Code to Automate the Design Of Coupled Cavities," presented at the XX International LINAC Conference, Monterey. CA, Aug 21-25, 2000, Los Alamos National Laboratory report LA-UR-00-3504.

[2] P. Smith, D. Christiansen, P. Greninger, and G. Spalek. "Automated Design of Coupled RF Cavities Using 2D and 3D Codes," these proceedings. 